\title{
German gynecologists' experience with a universal screening for gestational diabetes mellitus in daily practice: A qualitative study
}

\author{
Katharina Diehl ${ }^{1}$, Sven Schneider ${ }^{1}$, Christina Bock ${ }^{1}$, Holger Maul ${ }^{2}$, Helmut Kleinwechter ${ }^{3}$, Tatiana Görig ${ }^{1}$ \\ ${ }^{1}$ Mannheim Institute of Public Health, Social and Preventive Medicine, Medical Faculty Mannheim, \\ Heidelberg University, Mannheim, Germany \\ ${ }^{2}$ Department of Obstetrics, Marienkrankenhaus, Hamburg, Germany \\ ${ }^{3}$ Diabetologikum Kiel, Kiel, Germany
}

\section{Abstract}

Objective: In March 2012, a universal screening for gestational diabetes mellitus (GDM) was implemented in Germany. Despite international recommendations, a two-step approach was introduced [step 1: 50-g glucose challenge test (GCT); if GCT is suspicious, step 2 follows: 75-g oral glucose tolerance test with (OGTT)]. This qualitative study aimed at examining how gynecologists administer the screening for GDM in daily practice, whether they perceive any difficulties, and whether they have suggestions for improvement.

Material and Methods: Seventeen resident gynecologists were interviewed face-to-face in semi-structured interviews. The interviews were recorded, transcribed verbatim, coded, and analyzed using qualitative content techniques.

Results: We revealed differences in the screening administration. Three gynecologists directly offered the second step of the two-step screening (OGTT) instead of completing the first step before offering the second step. These gynecologists only conducted GCT if the woman (with statutory health insurance) was not willing to pay for OGTT. Critique concerns the late introduction of billing codes, lack of information from official institutions, unavailability of readymade syrup with 50 -g glucose, and lack of information material for pregnant women.

Conclusion: Our results reflect that not all gynecologists appear to conduct the screening conforming to the maternity directive. However, this has to be validated in larger quantitative surveys. That some gynecologists directly conducted OGTT may fuel the discussion regarding the screening procedure. The two-step approach was already highly controversial at the time of introducing the screening because national and international organizations recommend a one-step approach. Therefore, our results are also relevant for other countries who have implemented a two-step screening and for countries planning to implement a screening. (J Turk Ger Gynecol Assoc 2016; 17: 10-5)

Keywords: Gestational diabetes mellitus, glucose tolerance test, prenatal care, pregnancy, qualitative research

Received: 05 November, 2015

Accepted: 18 January, 2016

\section{Introduction}

Gestational diabetes mellitus (GDM) is associated with significant maternal and neonatal complications (1). In addition, it increases the mother's risk of developing a type 2 diabetes mellitus in long term (2). The prevalence of GDM in advanced economies ranges between $1.7 \%$ and $11.6 \%$ (3).

Because the prevalence is increasing worldwide (3), screening has been implemented in many countries worldwide. The screening aims at early detection and treatment of GDM. In particular, universal screening, i.e., an approach in which all pregnant women are screened for GDM regardless of their individual risk status, have been demonstrated to be cost-effective (4). Here, two different screening approaches are possible: GDM testing without previous screening (one-step approach) or a screening followed by definitive GDM diagnosis (two-step approach; 4).
In March 2012, a universal GDM screening was included in the German maternity directive. In contrast to the recommendations of the International Association of Diabetes and Pregnancy Study Groups (5), World Health Organization (6), German Diabetes Society, and German Society for Gynaecology and Obstetrics (7), a two-step approach was implemented. In the first step, every pregnant woman should be offered a glucose challenge test (GCT; 50-g glucose) as part of prenatal checkups. In the second step, an oral glucose tolerance test (OGTT; $75-g$ glucose) is offered for those with positive results in GCT.

As a result of the amendment in the maternity directive, all statutorily insured pregnant women (i.e., approximately 600.000 women/year) are entitled to GCT free of charge between gestational weeks $24+0$ and $27+6$. Reimbursement of the physicians is conducted by health insurances. When GCT is positive, the health insurances also pay for OGTT. If 
OGTT is performed without a verifiable reason, the pregnant woman has to pay for it herself. Before the introduction of this new guideline, gynecologists offered OGTT as a selfpaid prenatal check-up. The gynecologists decided to whom OGTT was offered. An exception is pregnant women who are privately insured. Here, the private health insurance pays for OGTT, even if no GCT was previously conducted.

To date, the management of the GDM screening in daily practice for German gynecologists remains unclear. In this study, resident gynecologists were offered the opportunity to comment on 1) administration of the screening in their practice, 2) potential difficulties perceived during screening administration, 3) reimbursement of the screening, 4) cooperation with diabetologists, and 5) potential suggestions for improvement of the screening procedure. Because this was the first time for German gynecologists to express their view in a scientific study, we selected a qualitative study design so as to not constrict the participants in providing their answers. In addition, this study design enabled us in obtaining a deeper knowledge of potential problems faced by resident gynecologists regarding the GDM screening.

\section{Material and Methods}

\section{Design}

In this qualitative exploratory study, semi-structured interviews with resident gynecologists were conducted to collect the data. This type of interview was selected to provide par- ticipants the opportunity to freely phrase their answers. This enables us to achieve a deeper understanding of the ideas, opinions, and worries of gynecologists (8), and thus, to identify potential snags in daily screening practice.

\section{Data collection}

Altogether, 50 randomly selected gynecologists in the German cities Mannheim, Ludwigshafen, and Heidelberg who were stratified by sex and number of gynecologists per city were contacted via a mail. Seventeen gynecologists agreed to participate in this study. The interviews were conducted face-to-face by the first author (KD, PhD, female sociologist) in the gynecologists' practice without the presence of a third person (Dec/02/2013Feb/06/2014).

We used a semi-structured interview guide with open-ended questions, which focused on the recent change in the German maternity directive. The interview guide was peer reviewed by experts in perinatology and obstetrics (HM) and diabetology (HK) and afterwards pre-tested in two interviews with gynecologists. These two interviews were included in the analysis because no essential changes in the interview guide were necessary.

Before the interview, the gynecologists were informed regarding the aim of the study and data protection. After the interview, sociodemographic and practice characteristics were collected. The average duration of the interviews was 33:12 min (minimum, $15 \mathrm{~min}$; maximum, $58 \mathrm{~min}$ ). They were audiotaped (Olympus DS-2500) and transcribed verbatim (191 pages).

\section{Table 1. Examples for quotations concerning the administration of the screening}

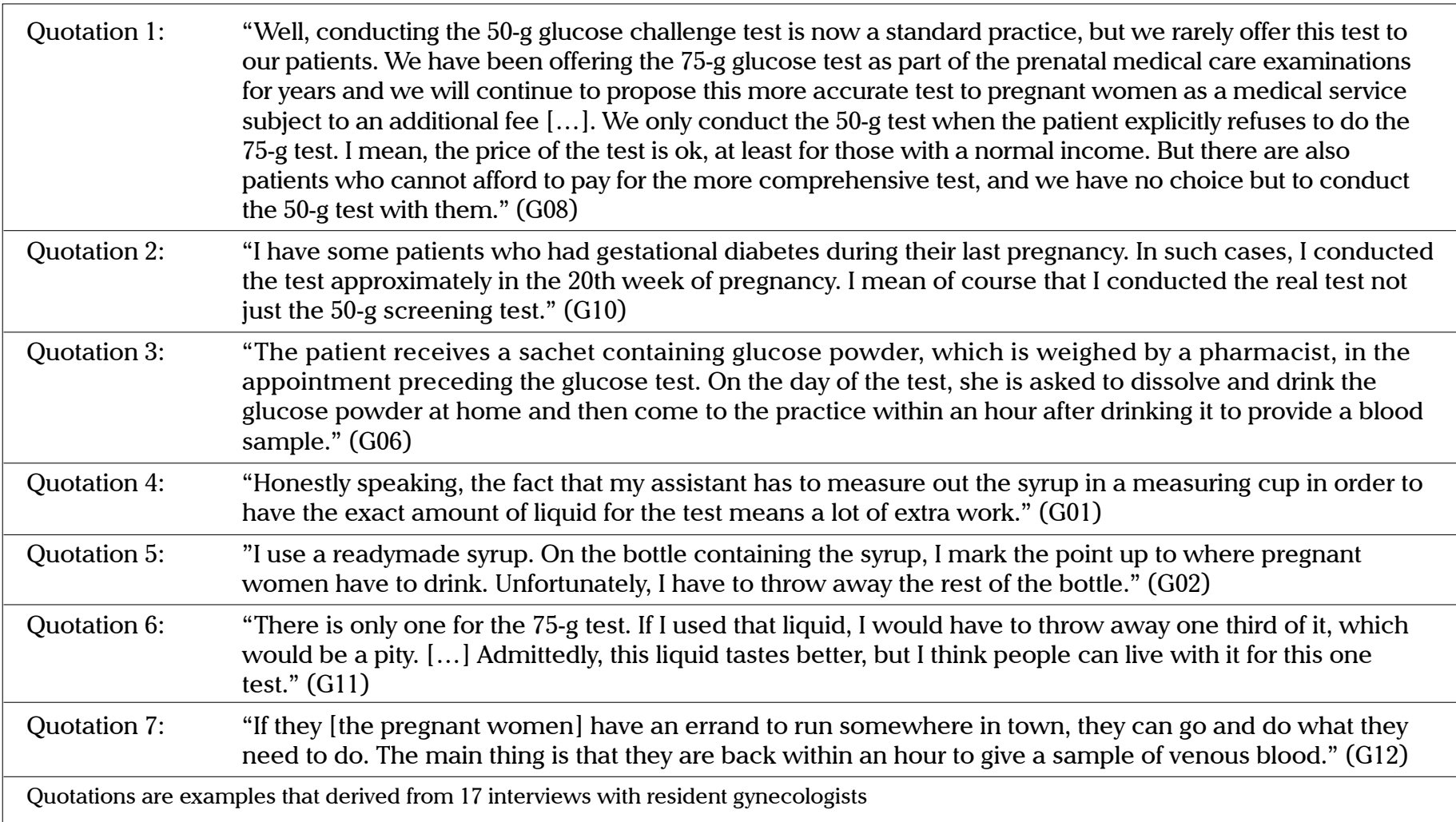




\section{Ethical consideration}

All gynecologists provided their written consent to participate. To reimburse them for the invested time, $80 €$ were paid. Approval of the Ethics Committee of the Medical Faculty Mannheim, Heidelberg University was obtained (2013-609N-MA).

\section{Participants}

The average age of the gynecologists was 52.9 years (minimum, 36 years; maximum, 70 years). They were resident for 13.9 years (minimum, 3 years; maximum, 29 years). Eleven of 17 gynecologists were female. The majority $(n=10)$ had a single practice.

\section{Analysis}

Qualitative content analysis following Mayring (9) was performed to identify themes, patterns, and contradictions by comparing the 17 interviews. Categories were identified by reviewing the data. Overall, seven categories comprising 22 codes were inductively developed to systemize the data. The data were independently coded by two researchers (KD and TG). The comparison revealed high agreement (89.9\%) (10). For coding the data, we used ATLAS.ti 7 (ATLAS.ti Scientific Software Development GmbH; Berlin, Germany). The results presented in this study are based on five categories (i.e., administration of the screening, potential difficulties perceived during screening administration, reimbursement of the screening, cooperation with diabetologists, potential suggestions for improvement of the screening procedure).

\section{Results}

\section{Administration of the screening}

Almost all participating gynecologists conducted the 50-g GCT to screen for GDM according to the recommendations set by the maternity directive. However, some participants considered GCT to be "not sensitive enough" (G13). Three gynecologists even recommended their patients to undergo the 75-g OGTT in the framework of the screening instead of GCT and allowed them to decide which test should be conducted (e.g., G08; quotation 1 in Table 1): Almost all participants $(n=15)$ were reported to perform the GDM screening within the time frame proposed by the German maternity directive, i.e., between gestational weeks $24+0$ and $27+6$. However, one gynecologist reported to offer GCT between gestational week 20 and 24 (G05). In some cases, gynecologists conducted GCT before week $24+0$ of pregnancy, e.g., because of irregularities during the prenatal check-ups. With regard to pregnant women at risk for developing GDM (e.g., women with GDM or macrosomia in preceding pregnancies), three interviewees stated that they directly offered them OGTT without previously conducting GCT (e.g., G10, quotation 2 in Table 1), which is in line with the recommended procedure. The majority of gynecologists $(n=12)$ used glucose powder to prepare the glucose solution for GCT (Figure 1). While in most cases the solution was prepared in the practice just before the conduct of the test, two gynecologists gave their patients a sachet containing glucose powder and had them prepare and drink the glucose solution at home prior to the gynecologist's appointment (e.g., G06; quotation 3 in Table 1). Limited capacities in the waiting room were named as the main reason for such a proceeding (G06).
Five gynecologists used the readymade glucose syrup that was reported to taste better and be easier for the patient to drink. In Germany, the syrup is only available in bottles containing 75-g glucose. This means that when the readymade syrup is used, the amount for GCT (50-g glucose) has to be manually measured. While some gynecologists conferred great importance for accurate measurement of the syrup (e.g., G01; quotation 4 in Table 1), some were less accurate in this regard (e.g., G02; quotation 5 in Table 1).

The fact that the syrup is only available in bottles with $75-\mathrm{g}$ glucose was the main reason for most participants preferring to use the glucose powder for GCT (e.g., G11; quotation 6 in Table 1).

Despite the recommended procedure, one gynecologist reported that he had his patients drink the glucose solution for GCT on an empty stomach (G05). In accordance with the guideline, the majority of participants stated that they had their patients stay in the practice after having drunk the glucose solution and sit still while waiting to provide a blood sample. However, some gynecologists conducted other examinations (e.g., cardiotocography) during the waiting time of 1 hour. Altogether, five gynecologists reported that their patients were allowed to move while waiting, e.g., walking to the practice for those who drank the liquid at home or just going for a short walk (e.g., G12; quotation 7 in Table 1).

Screening administration of the Glucose Challenge Test (GCT with 50 -g glucose)

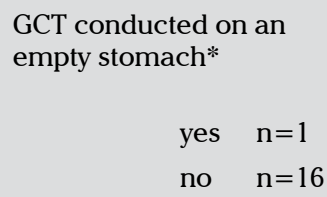
Type of glucose solution
used
GCT conducted with in the proposed time frame (between weeks $24+0$ and $27+6$ )

$$
\text { yes } \mathrm{n}=15
$$$$
\text { no } \mathrm{n}=1
$$

$$
\left.\begin{array}{ll}
\begin{array}{l}
\text { ready-made syrup } \\
\text { glucose powder }
\end{array} & \begin{array}{l}
n=10 \\
\mathrm{n}=2
\end{array} \\
\text { both } &
\end{array}\right\} \rightarrow \begin{aligned}
& \begin{array}{l}
\text { Prepation of glucose solu- } \\
\text { tion in the gynecologist's } \\
\text { practice }
\end{array} \\
& \begin{array}{l}
\text { yes } \\
n=10 \\
\text { no } n=2
\end{array}
\end{aligned}
$$

Proportion of pregnant women being screened regardless the screening test used (estimated by the gynecologists overlooking their own practice)

$\begin{array}{lr}\text { mean: } & 98 \% \\ \text { median: } & 100 \% \\ \text { min: } & 85 \% \\ \text { max: } & 100 \%\end{array}$

*GCT should not be conducted on an empty stomach according to the German maternity guideline

Figure 1. Information regarding the administration of the screening for gestational diabetes mellitus

Results based on 17 interviews with resident gynecologists. 
Compared with OGTT, GCT was considered to be "easier to conduct (for patients) because the patients do not have to be fasting and come in the morning" (G14). When asked for their opinion regarding how patients may perceive the screening test, most participants generally emphasized the positive aspects by saying, for instance, that pregnant women perceived GCT as a sign of better care (G02, G04, and G14) or as a meaningful measure "because in every family there is someone with diabetes" (G06). However, some interviewees also mentioned negative aspects by saying that some patients might have trouble providing blood samples (G02), investing extra time in conducting the test (G08), or with the taste of the glucose solution (G14, G16).

\section{Potential difficulties perceived during screening administration} Independent of whether GCT or OGTT was used for screening purposes, all gynecologists reported to conduct the test with nearly all of their patients. Many gynecologists explicitly emphasized that on the basis of the conventional understanding of screening measures, either of the test should be offered to all patients: "Strictly speaking, a screening includes everyone to determine those few who are affected. This is how every screening works." (G06). As a reason for non-administration of the screening test, participants most frequently named the patients themselves $(n=11)$. In this regard, two types of pregnant women were particularly problematic: those who missed the medical check-ups during the time frame when the GDM screening should be conducted and those who rejected the screening. However, most gynecologists emphasized that such patients were "absolute exceptions" (G04). One gynecologist who was resident in a district with a high proportion of migrants emphasized language problems with patients, which sometimes hampered the appropriate health education, and thus, the administration of the test: "Well you know, I have many patients who do not speak German as fluently as you would expect them to. In those cases, it is very hard to communicate what the test is all about and why it is so important" (G10).

Some gynecologists reported that some women experienced side effects during the test, such as nausea $(n=5)$, vomiting $(n=4)$, or circulatory complaints $(n=2)$. However, in general, the majority of participants stated that the conduct of the screening was unproblematic in this regard.

\section{Reimbursement of the screening}

One third of the gynecologists reported having had difficulties with the reimbursement of the screening test because the billing code for GCT in the doctors' fee schedule was approved more than 1 year after the GDM screening has been included in the maternity directive. Before this approval, the gynecologists had to submit the invoices to the health insurance and sometimes to "actively chase the payment" (G06), which was very time consuming. This shortcoming from the official institutions was sharply criticized by the gynecologists; for instance, "I personally find that from a health policy perspective, the introduction of such a regulation (the inclusion of the GDM screening in the maternity directive) should not be conducted with such poorly considered implementation strategies. If I want to introduce such a regulation, I should at least have the billing codes for the medical service in question and I should have calculated what costs to expect." (G16).
Some of the interviewees felt that they had not received sufficient information by the health insurance companies: "The health insurances offered us no help whatsoever (...) concerning the administration of the test." (G6). Therefore, every practice had to work out routines how to conduct the screening as efficiently and patient friendly as possible, which meant additional organizational efforts.

The majority of participants $(n=12)$ reported that with the introduction of the billing codes problems with the remuneration of the screening costs came to an end. Only one gynecologist mentioned that the remuneration of the costs for GCT and time spent for the patients' care during the administration of the test were not equal (G15).

\section{Cooperation with diabetologists}

Many gynecologists cooperated with several specialists. Approximately two-thirds of the gynecologists $(n=11)$ reported to cooperate with endocrinologists, eight with primary care physicians having an additional qualification in diabetology, and six with the women's hospitals. Most of the gynecologists $(n=9)$ reported having had positive experiences with diabetologists. However, some of the interviewees emphasized that despite their professional qualification, the diabetologists often had little experience with pregnant women (G02, G10, G14, and G16). From the gynecologists' point of view, the consequence of this inexperience could be that patients with diabetes do not receive optimal care, e.g., unnecessary measuring of blood glucose (G16) or missing the correct initiating point for insulin therapy (G13).

While all participants cooperated with diabetologists, differences existed with regard to the time point, i.e., as to when diabetologists were involved in the care of pregnant women. Approximately half of the gynecologists referred their patients to a diabetologist after positive OGTT, whereas the other half directly referred their patients to diabetologists with a positive result of GCT. Thus, the latter ones did not conduct OGTT themselves. A potential explanation was provided by G14: "As soon as (GCT) is suspected, any consecutive examination should be conducted by specialists. He or she should take over further tests because it is also him or her who will determine adequate glycemic control and therapy. It would be nonsense for the patient to undergo the 75 -g test in my practice and then again with the internist. Moreover, I would not want the colleague to base his glycemic control and therapy conclusions on my 75-g test either."

In general, gynecologists reported not having enough time to offer their patients in-depth nutrition counseling. This was another reason why they underlined the importance of diabetologists to counsel pregnant women regarding nutrition.

Potential suggestions for improvement of the screening procedure When asked regarding their potential suggestions to improve the screening, participants frequently mentioned the need for information that could be handed out to pregnant women. According to them, there is a lot of GDM information available on the internet for gynecologists; however, there is a lack of short and easy-to-understand information for patients (G02). Especially leaflets or brochures with some general information on GDM, with information on causes and consequences for mother and child (G17), and with information on the next 
steps after a positive screening (G15) were asked for. Two interviewees emphasized that it would be very helpful to have such information material in several languages (G11 and G16).

Moreover, participants underlined the need for more specific information for pregnant women, e.g., on how to eat healthy during the pregnancy (G15). However, another interviewee, who is very engaged in the field of GDM, stated that there is a lot of material regarding this topic available from various institutions (e.g., Federal Center for Health Education). One gynecologist emphasized that currently "the aspect of longterm importance (of GDM) for women is not sufficiently discussed. As soon as pregnancy is over, the topic of gestational diabetes disappears as well"(G16). In her opinion, during the pregnancy, "there is a very strong focus on the child, which is generally ok" (G16). However, according to her, there should be an equally strong focus on contraception and health behavior counseling for women after the pregnancy.

Another aspect in need of improvement from the gynecologists' point of view was the lack of readymade glucose syrup with 50-g glucose as described above. The fact that one-third of the bottle contents has to be thrown away if the gynecologist decides to use the readymade syrup with 75-g glucose for GCT was regarded as a "wastage" (G02). The availability of readymade glucose syrup was also considered necessary by gynecologists who used glucose powder. Some of them reported that the powder did not easily dissolve in water and emphasized that readymade syrup would be easier to drink for the patients because of its better taste.

\section{Discussion}

The majority of gynecologists adhered to the procedure mentioned in the maternity directive in terms of established parameters for GCT (i.e., gestational week, non-fasting, and no physical activity during waiting time). Nonetheless, contrary to the maternity directive, the $75-\mathrm{g}$ OGTT was frequently conducted as a screening test, particularly for privately insured pregnant women. However, there were also gynecologists who still offered OGTT for screening to every pregnant woman (not only privately insured) as a self-paid service and only conducted GCT, if the women were not willing to pay for OGTT. In addition, some gynecologists did not follow the Evidencebased Guideline on Diagnostics, Therapy, and Aftercare (11), which states that "During the test, the pregnant woman must sit near the test laboratory, shall not lie or move unnecessarily, and no other examination shall be carried out at this time." We found gynecologists that allowed pregnant women to walk (e.g., go shopping), handed out sachets containing glucose powder to pregnant women to dissolve and drink the glucose powder at home before coming to the practice, and conducted other examinations (e.g., cardiotocography) during the waiting time. Therefore, our results demonstrate that the GDM screening is not consistently conducted.

Perceived difficulties were the late introduction of billing codes and cooperation with diabetologists during the introduction period of the screening. Gynecologists stated that many diabetologists had a lack of experience with pregnant women. However, as time passed, this changed, and most gynecologists are now satisfied with the cooperation, particularly those working with endocrinologists.
Another issue that appeared during the interviews was that some gynecologists directly refer pregnant women to a diabetologist after a positive GCT, while other gynecologists only refer them after a positive OGTT. This means that some pregnant women might have to undergo three tests: GCT at the gynecologist's clinic, OGTT at the gynecologist's, and OGTT at the diabetologist's. This implies a high burden for the women and additional costs for the healthcare system.

While previous empirical studies focused on the experience of women having GDM (12), experience of midwives counseling women with GDM (13), and patients' attitudes towards the screening (14), this study is the first to deal with the daily practice of GDM screening from the gynecologists' point of view. The point in time for this study was ideal for two reasons. First, since the introduction of the screening, enough time has passed for the screening to be implemented into daily routine. Second, at the same time, the screening was still in an early stage; therefore, the gynecologists were able to remember the time of implementation and reorganization.

Nonetheless, there are several limitations that should be considered when interpreting our results. First, our data focus on the specific situation of the GDM screening in Germany and may not be applicable to other countries. However, our results may be interesting for countries who have implemented a two-step screening procedure. The difficulties in the interaction between gynecologists and diabetologists may also apply to other countries. Second, we cannot exclude a potential selection bias in our study population because the majority of participating gynecologists was well informed regarding the screening and conducted it by conforming to the maternity directive. Hence, our results may reflect a best-case scenario. Therefore, larger quantitative studies are required to obtain representative data. Our results revealed that the introduction of the GDM screening into the maternity directive did not go smoothly. In our study, those who conduct the screening as a daily routine, namely resident gynecologists, were questioned for the first time since the screening's introduction. We were able to reveal a number of critical points that had not been addressed to date. For example, gynecologists are to comply with the requirements set in the maternity directive, but at the same time, they are confronted with problems that make it difficult for them to meet these requirements. Among the problems raised, gynecologists particularly deplored the introduction period of the screening where they had to deal with financial uncertainty because of the lack of billing codes.

Following the gynecologists' suggestions, two aspects are highly important to facilitate the screening and patient counseling in the future. First, the provision of more information material covering issues such as the time after pregnancy and healthy nutrition in particular. Against the backdrop of their multiethnic clientele, the information material should be available in different languages. Second, the provision of readymade glucose syrup with 50 -g glucose would make the screening procedure more bearable for patients because of its better taste compared with glucose powder being dissolved in water. To date, there only exists readymade syrup with $75-\mathrm{g}$ glucose. This leads to a widespread use of glucose powder, which is less tolerated because of its extreme sweetness. 
One aspect that should be considered in future research is that the gynecologists are often not really involved in the screening administration. During the field phase of our study, it became apparent that often the medical technical assistant alone prepares the glucose solution, takes care of the women, and takes the blood sample. Informal talks with the assistants revealed that many pregnant women face difficulties drinking the glucose solution because they find it disgusting. Furthermore, it was mentioned that some pregnant women experience nausea (in particular, when it comes to drinking the solution that is not readymade but needs to be dissolved in water). Another difficulty, which was not mentioned by the gynecologists, appears to be the bad dissolution of the glucose powder in water. These facts reveal that the assistants have a different, possibly deeper insight into the conduct of the test. Therefore, it might be interesting to increase their involvement in future studies.

Our results are the first to shed some light into the "black box" GDM screening in gynecological care in Germany. After a difficult start, gynecologists finally succeeded in including the screening in their daily practice. However, we revealed differences in the administration of the screening. The different handling of the screening might be tackled by offering further training to the gynecologists or by proposing a more detailed procedure in the directive. Further studies, particularly quantitative representative studies, are urgently warranted for quality assurance of the screening conduction. Such studies would be useful to determine the proportion of gynecologists who conduct the screening according to the maternity directive (e.g., no conduct of GCT on empty stomach). Because of the qualitative nature of our study and a potential participation bias with more gynecologists, who are interested in this topic, participating in this study, we cannot provide an answer.

Nonetheless, our results imply that many pregnant women in Germany directly undergo OGTT despite the two-step approach that is suggested by the maternity directive. This might fuel the discussion on the two-step approach in Germany. Prior to the introduction of the screening, there were discussions on which screening should be included in the maternity directive. The German Diabetes Society and the German Society for Gynecology and Obstetrics preferred a one-step approach that offered OGTT to every pregnant women (7), which is in line with different international institutions $(5,6)$. Nonetheless, a more cost-efficient two-step approach was introduced after long discussions to have a less complex and less time-consuming test for all pregnant women in the first step. The results of our study are also interesting for other countries because of recent changes in statements and recommendations on the GDM screening worldwide.

Ethics Committee Approval: Ethics committee approval was received for this study from the ethics committee of Medical Faculty Mannheim, Heidelberg University (2013-609N-MA).

Informed Consent: Written informed consent was obtained from all gynecologists who participated in this study.

Peer-review: Externally peer-reviewed.

Author Contributions: Concept - K.D., C.B., S.V.S.; Design - K.D., C.B.; Funding - K.D., C.B.; Materials - K.D., T.G., S.V.S., H.M., H.K.; Data
Collection and/or Processing - K.D.; Analysis and/or Interpretation K.D., T.G.; Literature Review - K.D., T.G.; Writer - K.D., T.G.; Critical Review - C.B., H.M., H.K., S.V.S.

Acknowledgments: We would like to thank all gynecologists who were willing to take part in this study. Furthermore, we would like to thank Kristina Neumann for her support in transcribing the interviews.

Conflict of Interest: No author has any potential conflict of interest. Financial Disclosure: The study was funded by the young scientists program of the German network "Health Services Research BadenWürttemberg" of the Ministry of Science, Research and Arts in collaboration with the Ministry of Employment and Social Order, Family, Women and Senior Citizens, Baden-Württemberg.

\section{References}

1. HAPO Study Cooperative Research Group. Hyperglycemia and adverse pregnancy outcomes. N Engl J Med 2008; 358: 1991-2002. [CrossRef]

2. Kim C, Newton KM, Knopp RH. Gestational diabetes and the incidence of type 2 diabetes: a systematic review. Diabetes Care 2002; 25: 1862-8. [CrossRef]

3. Schneider S, Bock C, Wetzel M, Maul H, Loerbroks A. The prevalence of gestational diabetes in advanced economies. J Perinat Med 2012; 40: 511-20. [CrossRef]

4. Petrovic O. How should we screen for gestational diabetes? Curr Opin Obstet Gyn 2014; 26: 54-60. [CrossRef]

5. International Association of Diabetes Pregnancy Study Groups Consensus Panel. International association of diabetes and pregnancy study groups recommendations on the diagnosis and classification of hyperglycemia in pregnancy. Diabetes Care 2010; 33: 676-82. [CrossRef]

6. World Health Organization. Diagnostic Criteria and Classification of Hyperglycaemia First Detected in Pregnancy. Geneva: WHO; 2013.

7. Kleinwechter H, Schäfer-Graf U, Bührer C, Hoesli I, Kainer F, KautzkyWiller A, et al. Gestationsdiabetes mellitus (GDM) - Diagnostik, Therapie und Nachsorge. Diabetologie 2012; 7: 174-84. [CrossRef]

8. Flick U. An Introduction to Qualitative Research. London: SAGE Publications; 1998.

9. Mayring P. Qualitative Inhaltsanalyse: Grundlagen und Techniken [Qualitative content analysis: Basics and techniques]. Weinheim/ Basel: Beltz; 2010. [CrossRef]

10. Holsti O. Content analysis for the social sciences and humanities. Reading: Addison-Wesley; 1964.

11. Kleinwechter H, Schäfer-Graf U, Bührer C, Hoesli I, Kainer F, Kautzky-Willer A, et al. Gestationsdiabetes mellitus (GDM): Evidenzbasierte Leitlinie zu Diagnostik, Therapie und Nachsorge der Deutschen Diabetes-Gesellschaft (DDG) und der Deutschen Gesellschaft für Gynäkologie und Geburtshilfe (DGGG) [Gestational diabetes mellitus (GDM): Evidence based guideline for diagnostic, therapy and aftercare of the German Diabetes Society (DDG) and German Society for Gynecology and Obstetrics (DGGG)]. Kiel/ Stuttgart/Berlin: DDG, DGGG; 2011.

12. Devsam BU, Bogossian FE, Peacock AS. An interpretive review of women's experiences of gestational diabetes mellitus: proposing a framework to enhance midwifery assessment. Women Birth 2013; 26: e69-76. [CrossRef]

13. Persson M, Hornsten A, Winkvist A, Mogren I. "Mission impossible"? Midwives' experiences counseling pregnant women with gestational diabetes mellitus. Patient Educ Couns 2011; 84: 78-83. [CrossRef]

14. Griffiths RD, Rodgers DV, Moses RG. Patients' attitudes toward screening for gestational diabetes mellitus in the Illawarra area, Australia. Diabetes Care 1993; 16: 506-8. [CrossRef] 